\title{
High Resolution Transmission Electron Microscopy of Aluminum/Mo-Coated Glass Substrate Interface Bonded by Ultrasonic Wire Welding*
}

\author{
Chihiro IWAMOTO**, Shinobu SATONAKA**, Akio YOSHIDA***, \\ Tomoyuki NISHINAKA*** and Ken YAMADA*** \\ **Graduate School of Science and Technology, Kumamoto University \\ 39-1, Kurokami 2-chome, Kumamoto 860-8555, Japan \\ E-mail: iwamoto@mech.kumamoto-u.ac.jp \\ ***Toshiba Mitsubishi-Electric Industrial Systems Corporation \\ Mita 43 MT Bldg., 3-13-16 Mita, Minato-ku, Tokyo 108-0073, Japan
}

\begin{abstract}
Ultrasonic welding is an attractive joining method, because bonding is quickly obtained without extra heat or welding metal. Thus far, there have been several researches about the bonded interface structure and process of the ultrasonic welding. However, atomic structure analysis of the bonded interface was limited and sometimes controversial results were obtained. Besides, there were only few studies about application of the ultrasonic welding to $\mathrm{Al} / \mathrm{Glass}$ substrates and detailed bonding mechanism was unclear. In this report, the ultrasonic bonded interface between an $\mathrm{Al}$ wire and a Mo coated glass substrate was observed by high resolution transmission electron microscopy and the bonding mechanism was discussed. The bonded sample was cut perpendicular to the interface and, the atomic structure and chemical composition of the cross-section of the sample were analyzed. Grains in a Mo layer on the glass observed before bonding extended perpendicular to the glass substrate and terminated with nano sized pyramids. Structure of Mo grains remained unchanged after the bonding. Around the interface between $\mathrm{Al}$ and $\mathrm{Mo}$, several dislocations and $\mathrm{Al}$ sub-grains were produced in $\mathrm{Al}$ and no intermetallic compounds were observed. Al and Mo lattices were directly connected at the interface. This indicates that dislocations generated at the interface moved to form low angle grain boundaries during a recovery process without intermetallic formation. During the ultrasonic welding process, the pyramids of Mo surface were considered to work effectively to break a native oxide and produce the bonded interface.
\end{abstract}

Key words: Ultrasonic Welding, Nanostructure, Glass, HRTEM

\section{Introduction}

Ultrasonic welding is one of the popular methods to connect a metal to a metal or a metal to a ceramic using ultrasonic due to its productivity and handiness. Bonding was obtained without extra heat or a welding metal and it is widely studied in the field of the electric industry to connect wires to a circuit ${ }^{(1)}$.

Early TEM researches of the ultrasonic welding process suggested that the interface did not melt and there were no substantial diffusion during the bonding. Low density

*Received 20 July, 2011 (№. 11-0435) [DOI: 10.1299/jmmp.5.803]

Copyright (C) 2011 by JSME 
dislocation indicated that dynamic recovery or recrystallization occurred at the interface ${ }^{(2,3)}$. However, depending on the energy and bonding system, local melting at the bonded interface and intermetallic formation were also reported ${ }^{(4-7)}$.

Glass substrates were widely used in the various industrial fields and development of the joining technique between the glass substrate and metals is indispensable. Ultrasonic welding was successfully applied to the joining of metals to ceramics such as $\mathrm{Al}_{2} \mathrm{O}_{3}, \mathrm{SiO}_{2}$, $\mathrm{ZrO}_{2}, \mathrm{SiC}$, and $\mathrm{Si}_{3} \mathrm{~N}_{4}{ }^{(8)}$, and the application of the ultrasonic welding to the metal/glass substrate was also investigated ${ }^{(9,10)}$. However, no detailed analysis about the interface structure and bonding process at the metal/glass substrate has been carried out.

Recently, we obtained high strength joints between $\mathrm{Al}$ and various kinds of glass substrates using the ultrasonic welding. Tensile shear tests indicated that the joints were broken in the $\mathrm{Al}$ wire, and the interfaces or the glass substrates did not break. So far, three types of glass substrates, such as a Mo-coated glass substrate, an Ag-coated glass substrate and a normal glass substrate, were used for ultrasonic welding application. Among these glass substrates, the Mo-coated glass substrate and the Ag-coated glass substrate were more easily welded to Al than the normal glass substrate.

In this study, as the first steps to understand the high strength $\mathrm{Al} /$ glass joints formation process, the interface structure of the $\mathrm{Al} / \mathrm{Mo}$-coated glass substrate was observed using high resolution transmission electron microscopy (HRTEM). HRTEM is powerful tool to investigate a bonded interface to clarify the detailed bonding process. Thus far, we applied this method to several interfaces and clarified the atomic structure and the key factor to obtain the interface which have excellent properties ${ }^{(11,12)}$. Especially, in the study about the bonding process of brazing between ceramics and a molten alloy, we succeeded in observing the reaction process directly in-situ on an atomic scale ${ }^{(13,14)}$. The process of nucleation and growth of intermetallic compounds was dynamically revealed ${ }^{(15)}$.

Clarification of intermetallic compounds formation is one of the key factors to obtain high strength joints. Sometimes, intermetallic compounds formed at a bonded interface degrade mechanical properties of the joints ${ }^{(16)}$. At the interface between a circuit and wires, the intermetallic compounds also act as an extra resistance to a current. Using HRTEM, we investigated the bonded interface structure between $\mathrm{Al}$ and the Mo-coated glass substrate at an atomic level. Interface formation process between $\mathrm{Al}$ and the Mo-coated glass was also discussed.

\section{Experimental Procedure}

Specimens used were a pure $\mathrm{Al}$ foil with $110 \mu \mathrm{m}$ in thickness and a Mo-coated glass substrate (Geomatec Corporation). Mo was sputtered onto the glass which temperature was 100 degrees Celsius. The Al foil was bonded to the glass substrate by an ultrasonic wire welding machine (Toshiba Mitsubishi-Electric Industrial Systems Corporation). The welding energy was $8 \mathrm{~J}$.

The bonded specimen was sliced perpendicular to the bonded interface, which was followed by mechanical polishing and thinning using $\mathrm{Ar}^{+}$-ion beam under an accelerating voltage of $3 \mathrm{keV}$ for transmission electron microscope observation. Microstructures of the bonded specimen were observed using a FE-TEM (TECNAI F20, PHILIPS) at an accelerating voltage of $200 \mathrm{kV}$. Elements distribution around the interface was identified by energy-dispersive X-ray spectroscopy (EDS) equipped with the TEM. Electron beam diameter used for EDS was about $3 \mathrm{~nm}$.

\section{Experimental Results}

We observed the cross-section of the original glass substrate before observing the 
bonded sample to clarify the Mo structure on the glass substrate. Figure 1 is the bright field image of the Mo film coated on the glass. This image showed that thickness of the Mo layer covered the glass surface was about $150 \mathrm{~nm}$ and the Mo grains in the layer extended perpendicular to the glass substrate. Width of each grain was about 20 $\mathrm{nm}$ in average. Each grain terminated at the surface with a pyramid at the top. On the pyramids, almost no contamination or oxidized phases were observed. This means that the Al foil was bonded to this rough surface of Mo consisted of nano-pyramids during the ultrasonic welding.

In this study, we focused on the interface between $\mathrm{Al}$ and Mo to investigate the ultrasonic welding process, because the Mo layer was deposited before the ultrasonic welding, and the interface between Mo and glass did not break and relate to the mechanical properties of the joint.

Figure 2 is a low magnification bright

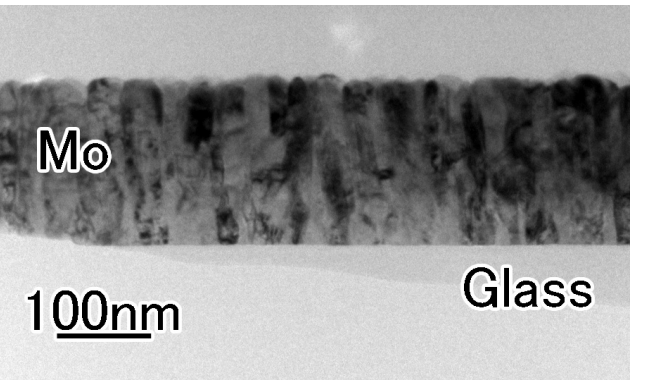

Fig.1 Bright field image of Mo layer coated on glass substrate

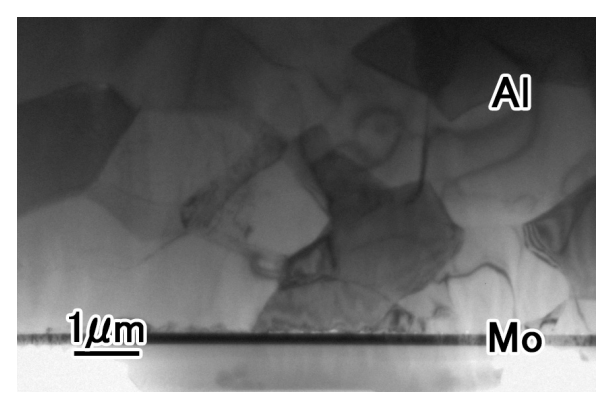

Fig. 2 Bright field image around interface between Mo coated glass and $\mathrm{Al}$

field image between $\mathrm{Al}$ and Mo with glass after the bonding. At this region, average diameter of the $\mathrm{Al}$ grains was about $2 \mu \mathrm{m}$. In the $\mathrm{Al}$ grains adjacent to the interface between $\mathrm{Al}$ and Mo, some strain contrasts were observed. Figure 3 is the one of the enlarged bright field images around the interface. In the Al grains, some dislocations were observed around the interface and contacted to the interface directly. Figure 3 suggested that the dislocations were generated at this interface during the ultrasonic welding.

In general, density of dislocations in a thin film observed by TEM is smaller than that in a bulk crystal. This is because of the thin film effect of the sample, which means that the sample used for TEM observation was too thin to keep the dislocations in the crystal, and optical conditions of the crystal direction to observe the dislocations affected on the imaging of the dislocations ${ }^{(17)}$. Then, actual density of the dislocations was thought to be higher than that observed in this image.

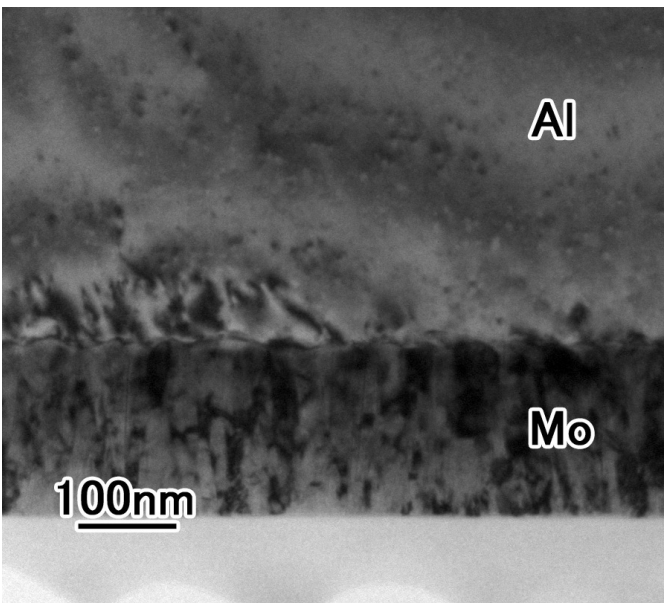

Fig. 3 Bright field image of interface between $\mathrm{Al}$ and Mo

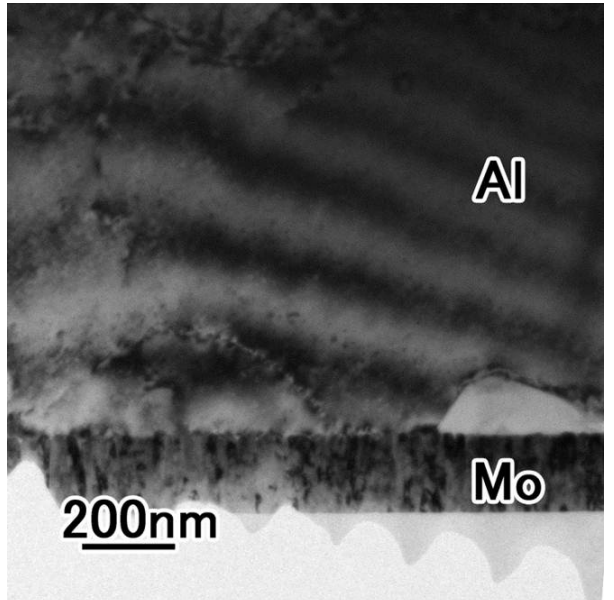

Fig. 4 Bright field image of interface between $\mathrm{Al}$ and Mo 
At the interface, Mo kept its characteristic pyramids at the top even after the ultrasonic welding in contrast to Al. Structure and, size and shape of the Mo grains were similar as those before the welding. This is because elastic modulus of Mo is 4 times higher than that of $\mathrm{Al}{ }^{(18)}$ and a fine-grained material is harder and stronger than that one that is coarse grained according to the Hall-Petch equation ${ }^{(19)}$.

At some regions adjacent to the Mo layer, small grains were observed in Al. Figure 4 is a bright field image of these small grains. These grains tended to extend parallel to the interface. Thicknesses of the grains were a few hundred nanometers and widths of

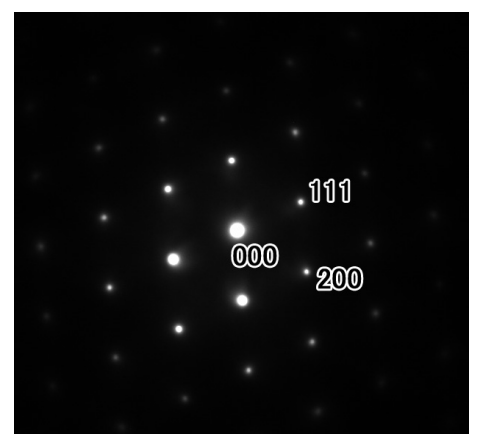

Fig. 5 Diffraction pattern taken from small grains adjacent to Mo the grains were about $500 \mathrm{~nm}$. In order to determine a kind of phase, crystallographic analysis and chemical composition analysis were made for these grains. Figure 5 is one of the typical diffraction patterns taken from the small grains. These diffraction patterns showed that the structure of the grain was Al and Fig. 5 indicated that the incident electron beam was parallel to the $<110>$ direction of Al. Surrounding Al and the other small grains also showed $<110>$

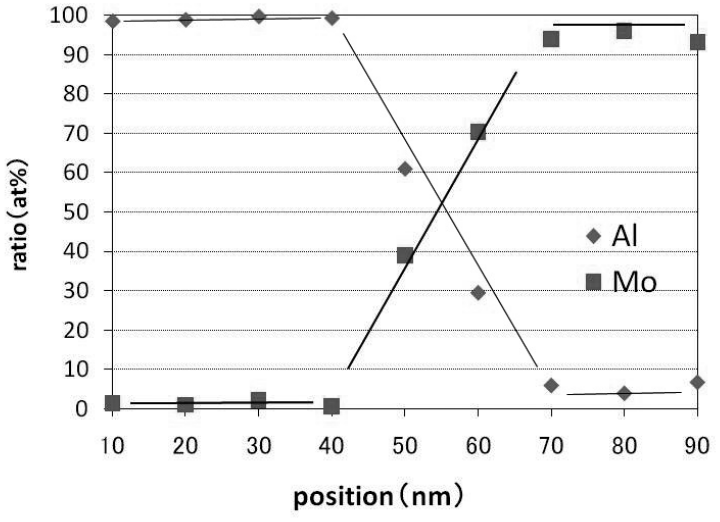

Fig. $6 \mathrm{Mo} / \mathrm{Al}$ element ratio perpendicular to interface between $\mathrm{Mo}$ and $\mathrm{Al}$

diffraction patterns with small deviations $\left(<15^{\circ}\right)$. This means that the interfaces between small grains and surrounding Al were small angle grain boundaries.

Distribution of the chemical elements was clarified by EDS profile taken perpendicular to the interface between Mo and Al with $10 \mathrm{~nm}$ intervals. After quantification calculation, ratio of $\mathrm{Al}$ and Mo was plotted in a graph (Fig. 6). This graph indicated that the ratio of $\mathrm{Al}$ was constant and almost $100 \%$ in the Al-rich region and at the interface, gradually reduced to the Mo-rich region. The ratio of the Al-rich region confirmed that the small grains were Al. At the interface, the ratio of the Mo and $\mathrm{Al}$ gradually changed. However, no plateau in the ratio variation, which suggests existence of the intermetallic compound area, was found in the transition region. Width of this composition transition region was about $20 \mathrm{~nm}$, which was nearly the same as the height of the Mo pyramids. This means that the variation of the Mo ratio indicated the volume ratio of the Mo pyramid in the Al film through the electron beam direction. Thus, from chemical

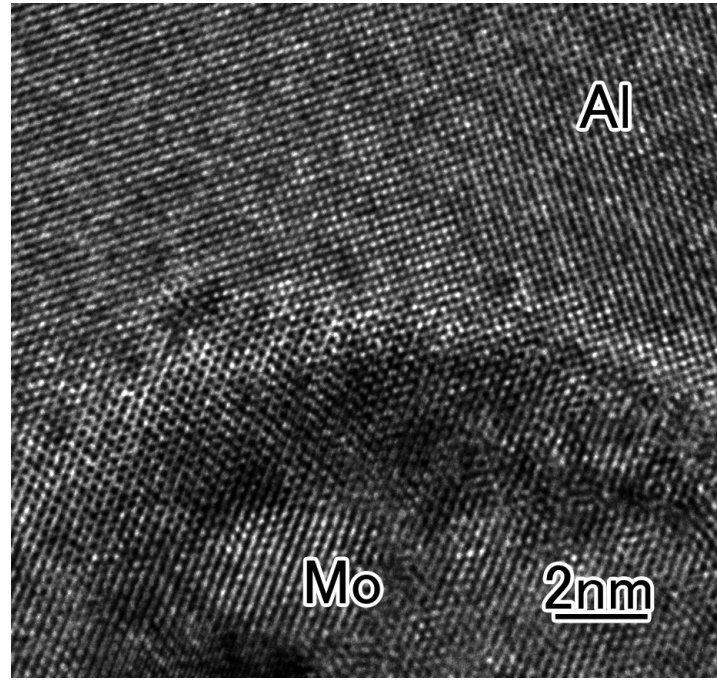

Fig. 7 High resolution image of interface between $\mathrm{Al}$ and Mo 
analysis, we did not obtain the evidence of the intermetallic compound formation.

Furthermore, high resolution images at the interface between $\mathrm{Al}$ and Mo were taken to clarify the detailed structure. Figure 7 is a typical high resolution image of the interface. Incident electron beam was parallel to the [100] direction of Al. Lattice fringe in Mo corresponded to the (110) plane. At the interface, Mo lattice directly connected to $\mathrm{Al}$ lattice and lattices of intermetallic compounds were not observed. Together with the result of the chemical analysis, it was concluded that the intermetallic compounds did not form during the ultrasonic welding under present

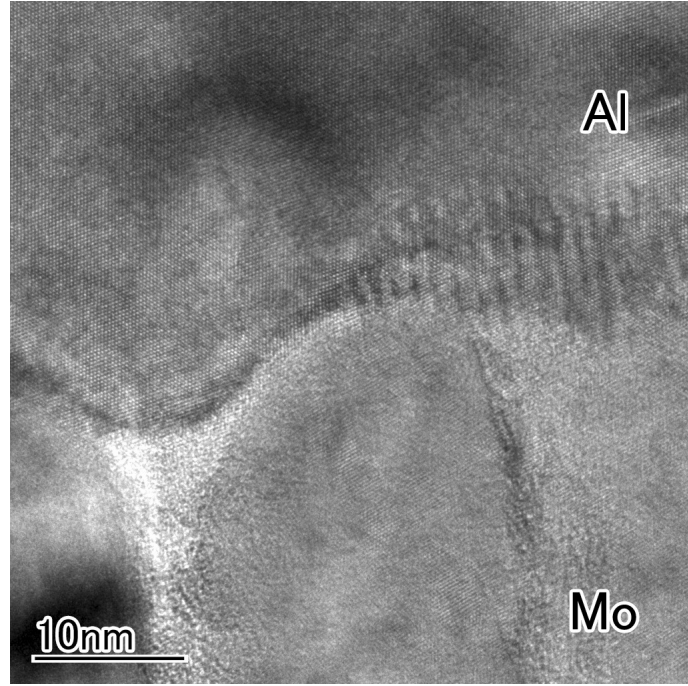

Fig. 8 High resolution image around interface between $\mathrm{Al}$ and $\mathrm{Mo}$ experimental conditions.

With direct bonded region, amorphous phase was observed at some regions around the interface. Figure 8 is a high resolution image of the amorphous region. There were three Mo grains extended perpendicular to the interface in Fig. 8. At the top, Mo grains directly connected to $\mathrm{Al}$ and the amorphous phase presented between the Mo grains. Width of the amorphous region decreased, as the distance from the interface increased, and finally, the amorphous region disappeared to be a Mo grain boundary. This amorphous region was considered to be a native oxide layer or contaminants existed on the specimen surface before bonding.

\section{Discussion}

Around the interface, dislocations and small grains were observed. However, vortices or swirls of Al structure, which appeared in high power ultrasonic spot welding ${ }^{(20)}$ were not observed. In this experiment, welding energy of the ultrasonic welding was only $8 \mathrm{~J}$ and quite smaller than that of the high power ultrasonic welding (over hundreds $\mathrm{J}$ ). It was not enough to deform the welded interface dramatically in this experiment. Strain contrast was observed in Al within the region about $200 \mathrm{~nm}$ in width adjacent to Mo and Mo did not change its structure.

Dislocations observed in the present experiments were considered to be generated by the pyramids observed at the top of the Mo grains during the ultrasonic welding. In general, dislocations were frequently observed around the ultrasonic welded interface, because of the plastic deformation during the bonding ${ }^{(2,3,6)}$. In the ultrasonic welding, ultrasonic also activated dislocations and increased the dislocation density ${ }^{(7)}$. In addition, in the present study, the characteristic structure of Mo would become a resistance to sliding between Al and the Mo coated glass substrate during the ultrasonic welding and act as a source of dislocation generation due to stress concentration.

From the crystallographic and chemical analysis, small grains at the interface were $\mathrm{Al}$ sub-grains. There were no intermetallic compounds around the interface. Several papers suggest that temperature at the interface during the ultrasonic welding was well below the melting temperature ${ }^{(20,21)}$ of samples. Then, a recovery process occurred during bonding process by dislocation movement to form low angle grain boundary without intermetallic formation. Our observation was in good agreement with the previous TEM observation ${ }^{(2)}$. 
Sometimes, lack of resolution of the structure observation gave controversial results about the intermetallic formation and local melting around the interface during welding ${ }^{(4)}$. However, present high resolution observations gave the atomic information around the interface, then, it provided the conclusive evidence of the $\mathrm{Al} / \mathrm{Mo}$ direct bonded interface without intermetallic formation under present experimental conditions.

It was indicated that surface contaminants and native oxides, which existed before bonding and were believed to prevent contact and bonding between bonded materials, were dispersed during ultrasonic welding ${ }^{(21)}$ and remained around the interface ${ }^{(2)}$. These contaminants were observed between the Mo grains in the present experiments. Our observation suggested that the pyramid of the Mo grains was an effective shape to break the surface contaminants and oxides, and remove them from the contacting area. Top of the Mo pyramids connected to $\mathrm{Al}$ to produce microbonds, as observed in Fig. 8. Because the effective net area of these microbonds related to the joint strength ${ }^{(20)}$, Mo pyramids would contribute to obtain the high strength $\mathrm{Al} /$ Glass substrate joints.

In the 5052/SPCC system, an amorphous phase produced at the interface and contributed to the good bonding ${ }^{(22)}$. Detailed analysis of the amorphous phase observed in this study and relationship between the amorphous phase and mechanical properties of the joint are our future work.

\section{Summary}

Interface between $\mathrm{Al}$ and Mo-coated glass substrate bonded by ultrasonic welding was observed using HRTEM. Surface of the grains in Mo layer had nano sized pyramids at their top. Structure of Mo extended perpendicular to the glass substrate remained unchanged after bonding. Around the interface between $\mathrm{Al}$ and Mo, several dislocations and Al sub-grains were produced in $\mathrm{Al}$ and no intermetallic compounds were observed. Al and Mo lattices were directly connected at the interface. Pyramids on Mo were considered to work effectively to break the native oxide and produce the bonded interface.

\section{References}

(1) Murali, S., Srikanth, N., and Vath III, C. J., 2003, "Grains, deformation substructures, and slip bands observed in thermosonic copper ball bonding," Mater. Charact., 50(1), pp. 39-50. (2) Krzanowski, J. E., 1990, "A Transmission Electron Microscopy Study of Ultrasonic Wire Bonding," IEEE Transactions on Components, Hybrids, and Manufacturing Technology.

(3) Murdeshwar, N., and Krzanowski, J. E., 1997, "A Microstructural Study of Dislocation Substructures Formed in Metal Foil Substrates during Ultrasonic Wire Bonding," Metallurgical and Materials Transactions A, 28A, pp. 2663-2671.

(4) Brodyanski, A., Born, C., and Kopnarski, M., 2005, "Nm-scale resolution studies of the bond interface between ultrasonically welded Al-alloys by an analytical TEM: a path to comprehend bonding phenomena?," Appl. Surf. Sci., 252(1), pp. 94-97.

(5) Geissler, U., Schneider-Ramelow, M., Lang, K.-D., and Reichl, H., 2006, "Investigation of Microstructural Processes during Ultrasonic Wedge/Wedge Bonding of AlSil Wires," J. Electron. Mater.

(6) Karpel, A., Gur, G., Atzmon, Z., and Kaplan, W. D., 2007, "TEM microstructural analysis of As-Bonded Al-Au wire-bonds," Journal of Materials Science, 42(7), pp. 2334-2346.

(7) Li, J., Han, L., Duan, J., and Zhong, J., 2007, "Microstructural characteristics of Au/Al bonded interfaces," Mater. Charact., 58(2), pp. 103-107.

(8) Matsuoka, S.-i., 1994, "Ultrasonic welding of ceramic/metal," J. Mater. Process. Technol., 47(1-2), pp. 185-196.

(9) Kuckert, H., Born, C., Wagner, G., and Eifler, D., 2003, "Residual Stress Distributions in Glass/Metal-Joints produced by Ultrasonic Torsion Welding," Mat.-wiss. u. Werkstofftech, 
34 , pp. 30-33.

(10) Matsuoka, S.-i., Yamazaki, H., and Ishikuro, T., 2008, "Direct Welding of Alminum/Glass by Ultrasonic Vibration," Transactions of Japan Society of Mechanical Engineers.

(11) Iwamoto, C., Yang, H. S., and Yoshida, T., 2002, "Direct growth of c-BN on a mono-structured transition layer by plasma-enhanced chemical vapor deposition," Diamond Relat. Mater., 11, pp. 1854-1857.

(12) Iwamoto, C., Satonaka, S., Yamamoto, T., Ikuhara, Y., and Matsuhata, H., 2006, "1 A-resolution chemical imaging by phase contrast technique," J. Appl. Phys., 99, p. 104909.

(13) Iwamoto, C., and Tanaka, S.-I., 1998, "Reactive Wetting of Ag-Cu-Ti on $\mathrm{SiC}$ in HRTEM," Acta Mater., 46(7), pp. 2381-2386.

(14) Iwamoto, C., and Tanaka, S.-I., 2010, "In-situ HRTEM Observations of Spreading Reactive Molten Alloy on Ceramic Substrates," Advances in Science and Technology, 64, pp. 83-87.

(15) Iwamoto, C., Ichinose, H., and Tanaka, S.-I., 1999, "Atomic Observation at the Reactive Wetting Front on SiC," Philos.Mag.A, 79(1), pp. 85-95.

(16) Qiu, R., Iwamoto, C., and Satonaka, S., 2009, "In situ scanning electron microscopy observation of fracture crack propagation in the welding interface between aluminium alloy and steel," Mater. Sci. Technol., 25(10), pp. 1189-1192.

(17) Hirsch, P., Howie, A., Nicholson, R., Pashley, D. W., and Whelan, M. J., "Electron Microscopy of Thin Crystals," Krieger Publishing Company.

(18) Boyer, H. E., and Gall, T. L., 1985, Metals Handbook, American Society for Metals.

(19) William D. Callister, J., 2007, Materials Science and Engineering an Introduction, John Wiley \& Sons, Inc.

(20) Bakavos, D., and Prangnell, P. B., 2010, "Mechanisms of joint and microstructure formation in high power ultrasonic spot welding 6111 aluminium automotive sheet," Mater. Sci. Eng., A, 527(23), pp. 6320-6334.

(21) Siddiq, A., and Ghassemieh, E., 2008, "Thermomechanical analyses of ultrasonic welding process using thermal and acoustic softening effects," Mech. Mater., 40(12), pp. 982-1000.

(22) Hamed, A.-A., Katoh, M., Nishio, K., Yamaguchi, T., and Tsue, Y., 2005, "Evaluation by Ultrasonic Testing and TEM Observation of Bond Interface for Ultrasonic Bonds of 5052/SUS304 and 5052/SPCC," Journal of the Japan Welding Society. 